\begin{tabular}{|c|c|c|c|c|}
\hline & Training Dataset & $\begin{array}{c}\text { Validation } \\
\text { Dataset }\end{array}$ & Testing Dataset & $\begin{array}{c}\text { Total } \\
\text { (frames) }\end{array}$ \\
\hline Number of patients & 107 & 14 & 78 & 199 \\
\hline Adenomatous polyp & 122 & 19 & 94 & $\begin{array}{c}235 \\
(21,831)\end{array}$ \\
\hline Hyperplastic polyps & 21 & 2 & 35 & $\begin{array}{c}58 \\
(3,262)\end{array}$ \\
\hline Sessile serrated lesions & 43 & 11 & 23 & $\begin{array}{c}77 \\
(6,017)\end{array}$ \\
\hline $\begin{array}{l}\text { Traditional serrated } \\
\text { adenomatous polyps }\end{array}$ & 1 & 0 & 0 & $1(42)$ \\
\hline $\begin{array}{c}\text { Total number of polyps } \\
\text { (frames) }\end{array}$ & $\begin{array}{c}187 \\
(14,936)\end{array}$ & $\begin{array}{c}32 \\
(1,896)\end{array}$ & $\begin{array}{c}152 \\
(14,320)\end{array}$ & $\begin{array}{c}371 \\
(31,086)\end{array}$ \\
\hline
\end{tabular}

diagnose adenomas and a specificity of $90 \%$. The CNN achieved an area under the curve (AUC) of $97 \%$. On a per polyp analysis, the accuracy of the CNN characterisation was $92 \%$, with a sensitivity of $92 \%$ and a specificity of $93 \%$.

Conclusion The largest annotated dataset of NBI polyp images has been collated for the training and evaluation of artificial intelligence to support optical diagnosis. This work demonstrated the capability of AI to differentiate adenomatous from non-adenomatous polyps in-vitro, with a high level of accuracy.

\section{HTU-2 EUS GUIDED GASTROJEJUNOSTOMY FOR THE MANAGEMENT OF GASTRIC OUTLET OBSTRUCTION: A SINGLE CENTRE EXPERIENCE}

Wei On*, Matthew T Huggett, Bharat Paranandi. Leeds Teaching Hospitals NHS Trust, Leeds, UK

\subsection{6/gutjil-2021-BSG.49}

Introduction There are various modalities of management of gastric outlet obstruction (GOO) namely endoscopic enteral stenting, surgical bypass or placement of a venting gastrostomy tube. Endoscopic ultrasound guided gastrojejunostomy (EUSGJ) via placement of a lumen apposing metal stent has recently emerged as a viable and effective method of managing GOO. We aimed to describe the procedural characteristics, technical and clinical outcomes in patients who underwent EUS-GJ at our institution.

Methods A prospectively collected database of consecutive EUS-GJ procedures from August 2018 to October 2020 at our institution was reviewed retrospectively. All procedures were performed by two expert pancreatobiliary endoscopists. Recorded variables included patient demographics, technical success, clinical success, adverse events, 30-day all cause mortality and follow-up duration.

Results Sixteen patients $(9$ males) with a mean age of 64.5 years old (range 48 - 80 years old) were identified. Malignancy was the predominant aetiology of GOO in our patient cohort $(81.3 \%, \mathrm{n}=13)$. The technical success rate (defined as fluoroscopic and endoscopic confirmation of adequate stent deployment and positioning) was $93.8 \%(\mathrm{n}=15)$, in whom the clinical success rate (defined as toleration of at least liquid diet without vomiting before discharge) was $100 \%$. In the solitary case of technical failure, dislodgement occurred during balloon dilatation of the stent, the defect was endoscopically closed with clips and a duodenal stent was placed. The patient did not experience any adverse consequences post-procedure. Nine patients who required intravenous parenteral nutrition pre-procedure were successfully weaned off following EUS-GJ.
The median follow-up was 109 days (range 5 - 383 days). No adverse events were encountered. One patient required reintervention at day 70. 30-day all cause mortality was 6.3\% $(\mathrm{n}=1)$ and the death was due to malignant disease progression.

Conclusions EUS-GJ is an effective and safe procedure for the management of GOO. It should be considered in appropriately selected patients if there is available expertise.

\section{HTU-3 OPTICAL DIAGNOSIS OF SMALL POLYPS AT COLONOSCOPY VERSUS HISTOPATHOLOGY: MOVING TOWARDS A NEW GOLD STANDARD?}

${ }^{1}$ Ahmir Ahmad*, ${ }^{1}$ Ana Wilson, ${ }^{2}$ Morgan Moorghen, ${ }^{1}$ Angad Dhillon, 'Siwan ThomasGibson, 'Noriko Suzuki, ${ }^{1}$ Adam Humphries, ${ }^{1}$ Adam Haycock, ${ }^{1}$ Kevin Monahan, ${ }^{1}$ Margaret Vance, 'Brian Saunders. 'Wolfson Unit for Endoscopy, Imperial College London, London, UK; ${ }^{2}$ Pathology Department, St Mark's Hospital, London, UK

\subsection{6/gutjnl-2021-BSG.50}

Introduction Histopathology is regarded as the gold standard for diagnosis of small colonic polyps. However, there is growing interest in optical diagnosis and implementation of a 'resect and discard' strategy. Our aim is to evaluate accuracy of histopathology reporting where a high confidence diminutive polyp optical diagnosis was made and to assess the impact of performing additional tissue section re-cuts, where there is a discrepancy.

Methods Eight bowel cancer screening colonoscopists optically diagnosed 639 diminutive polyps during the period Feb-Nov 2020 in the early phase of a prospective feasibility study of optical diagnosis (DISCARD3). Each polyp diagnosis was evaluated by the colonoscopist as high or low confidence. All retrieved polyps were sent for histopathology.

Discrepancy between high confidence optical diagnoses and histopathology were re-reported by a second pathologist blinded to the original optical and histological call. If discrepancy remained after re-review, the polyp was re-cut into deeper levels and a third blinded histopathology review performed.

Results Of 639 diminutive polyps, 468 (73.2\%) were high confidence optical calls and 171 (26.8\%) were low confidence (see figure 1).

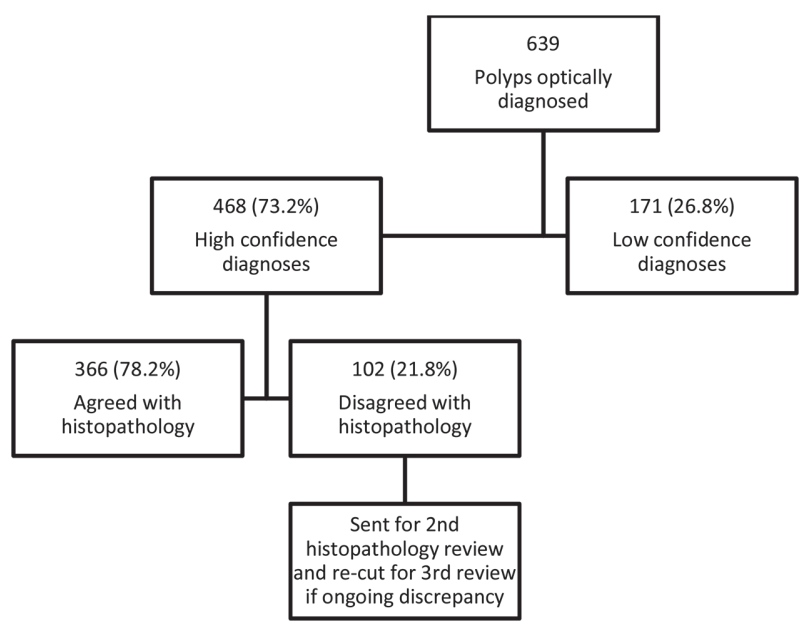

Abstract HTU-3 Figure 1 Overview of study 